(C) 2017 IEEE. Personal use of this material is permitted. Permission from IEEE must be obtained for all other uses, in any current or future media, including reprinting/republishing this material for advertising or promotional purposes, creating new collective works, for resale or redistribution to servers or lists, or reuse of any copyrighted component of this work in other works. 


\title{
DISCONTINUOUSLY OPERATED MOX SENSORS FOR LOW POWER APPLICATIONS
}

\author{
Javier Burgués ${ }^{1,2, *}$, Jordi Fonollosa ${ }^{1,2}$, Santiago Marco ${ }^{1,2}$
}

${ }^{1}$ Department of Engineering: Electronics, Universitat de Barcelona, Marti i Franqués 1, 08028-

Barcelona, Spain

2 Signal and Information Processing for Sensing Systems, Institute for Bioengineering of Catalonia, Baldiri Reixac 4-8, 08028-Barcelona, Spain

*Correspondence: jburgues@ibecbarcelona.eu; Tel.: +34 934031118

\section{ABSTRACT}

Metal oxide semiconductor sensors are limited by their low selectivity, high power consumption and temporal drift. This paper proposes a novel discontinuous temperature modulation operation mode characterized by on-demand measurements and periodic warm-up cycles. The performance of two sets of FIS SB-500-12 sensors, one group continuously operated and the other group discontinuously operated, was compared in a scenario of carbon monoxide detection at low concentrations for five consecutive days. Results showed that the discontinuous operating mode moderately increased the prediction error and the limit of detection but was advantageous in terms of energy savings (up to $60 \%$ with respect to the continuous temperature modulation mode).

Index Terms - Low power, MOX sensors, temperature modulation, discontinuous operation, duty-cycling.

\section{INTRODUCTION}

Metal oxide semiconductor (MOX) sensors are known for their sensitivity to a large variety of gases, low cost and small size. However, their main limitations are lack of selectivity, high power consumption and temporal drift [1]. The high power demands derive from the current injected to the heating resistor used to increase the temperature of the sensing layer for more efficient chemical reactions. It is well known that temperature modulation increases the selectivity compared to isothermal operation [2]. In temperature modulation, the sensor surface is periodically heated up and cooled down by applying an arbitrary waveform to the heater voltage. In the simplest case, an asymmetric square waveform that commutes between two heater voltages is used (see Fig. 1). The conductance vs. time waveform is characteristic of the analyte gas and its concentration. Longer cycles of the heating function sharpen the characteristic features of the conductance pattern at the cost of longer measurement intervals (a prediction is obtained after each temperature modulation cycle). To reduce temporal drift and

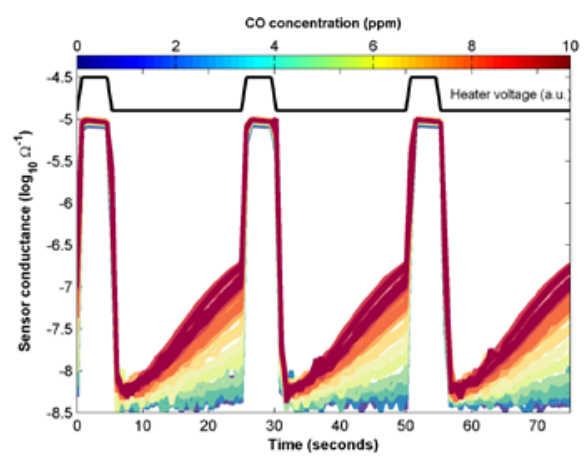

Figure 1. Conductance of a temperature modulated sensor during three consecutive heating cycles. The 66 measurements corresponding to one experimental day are overlapped. The color indicates the $\mathrm{CO}$ concentration.

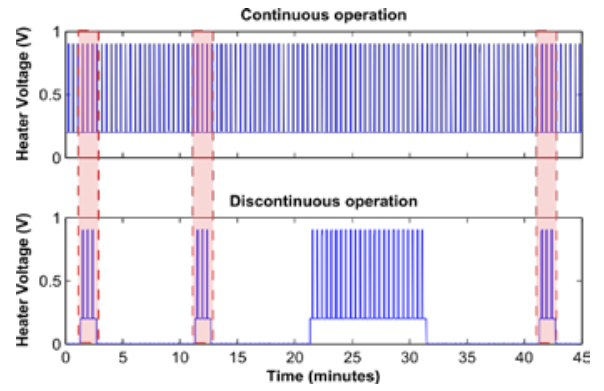

Figure 2. Heater voltage waveform in continuous and discontinuous mode. Each shaded area represents a single measurement (see Fig. 1 for an enlarged view). The heating cycles from $t=22$ to $t=32$ minutes, in the discontinuous mode, correspond to a warm-up block.

increase the repeatability of measurements, the sensors must be continuously powered, even if no measurements are being 
taken [3]. Because continuously powering a MOX sensor is energy costly (typically hundredths of $\mathrm{mWh}$ ), several studies have attempted at minimizing power consumption by means of duty-cycling. Duty-cycling saves energy by periodically switching on and off the sensor power. The duty cycle is the percentage of time during which power is applied with respect a cycle period. Oletic et al. [4] found that a train of 6 pulses of $30 \%$ duty cycle and $1 \mathrm{~s}$. period was enough warmup after a $30 \mathrm{~s}$. shutdown. Jelicic et al. [5] reported a duty cycle of $20 \%$, period of $1 \mathrm{~s}$. and a measurement time of only $65 \mathrm{~ms}$. Sayhan et al. [6] reduced the duty cycle to $10 \%$ in 1-minute pulses. Rossi et al. [7] used a $0.5 \%$ duty cycle and a period of 15 minutes. Recently, Macías et al. [8] proposed applying a $8.3 \%$ duty cycle in 10 minute cycles.

A common factor between the previous works is that measurements are assumed to be taken periodically. While this might be the case in many applications, in some situations the measurements are taken on-demand. This means that the sensor is switched off for a long period of time (days, weeks or even months) and a measurement can be requested at any moment. For example, an ethanol MOXbased breath analyzer might be powered on only to check the blood alcohol content in the driver's blood. Vergara et al. [9] proposed this kind of scenario but the amount of time the sensors were disconnected was not specified.

Motivated by this type of applications, the current work explored the behavior of discontinuously operated MOX sensors in a scenario of on-demand measurements of carbon monoxide (CO). The prediction quality, Limit of Detection (LOD), measurement time and short term drift were compared to a set of sensors continuously powered.

\section{MATERIALS AND METHODS}

A board including 14 sensors of the same type (SB-500-12, provided by FIS) and the corresponding read-out electronics was built. The sensors were warmed up for one week before the experiments. The heater voltage was modulated in the range $0.2-0.9 \mathrm{~V}$ following manufacturer recommendations. Half of the sensors were operated in a continuous mode whereas the remaining sensors were discontinuously operated. In the latter case, the elapsed time between measurements was a random variable in the range $[10,60]$ minutes. To increase the repeatability of measurements and reduce temporal drift, the discontinuously operated sensors were heated up for $10 \mathrm{~min}$. whenever the off-time period exceeded 30 min. (see Fig. 2). A single measuremen consisted on three consecutive heating periods that lasted 75 s. (see Fig. 1). In this configuration, the discontinuously operated sensors were powered on during 24 minutes each hour, which represents energy savings of $60 \%$ with respect to the continuous mode.

The sensor board was placed in a gas chamber $\left(250 \mathrm{~cm}^{3}\right.$ internal vol.) in which dynamic gas mixtures of $\mathrm{CO}$ and synthetic air were randomly introduced at a constant flow rate of $480 \mathrm{ml}_{\mathrm{n}} / \mathrm{min}$ for 10 minutes. The experimental concentrations ranged from 0 to $10 \mathrm{ppm}$ in steps of $1 \mathrm{ppm}$

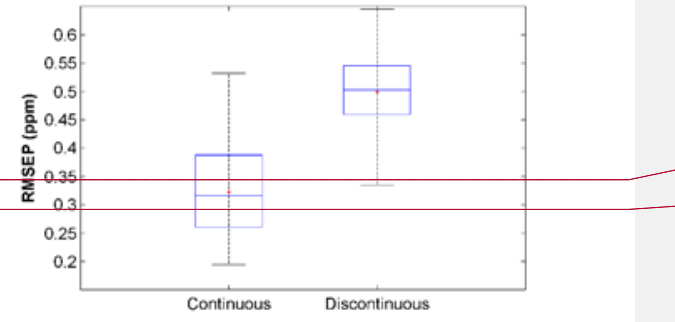

Eliminado: the

Eliminado: on over one
Figure 3. Box plots of intraday RMSEP for continuously and discontinuously operated sensors in all experimental days.

Six repetitions were done for each concentration. Therefore, 66 measurements were acquired in each experiment, which lasted 22 hours and was replicated on four consecutive days.

Partial Least Squares (PLS) calibration models were built using logarithmic sensor conductance patterns (see Fig. 1 ), cropped from $t=0$ to $\{5,10,15, \ldots, 75\}$ s. to simulate several measurement times. The dataset was split into calibration and test samples. The calibration samples were taken from the even concentration levels $(0,2,4,6,8,10 \mathrm{ppm})$ and the test set was formed by the odd concentration levels $(1,3,5,7,9 \mathrm{ppm})$. The number of latent variables (LV) was optimized by inspection of the root mean squared error in cross validation (RMSECV) vs. number of LV curve, calculated via bootstrapping on the calibration samples. Two types of external validation were performed: intraday and interday. In the former case, the models calibrated on day $d$ were tested against test samples of day $d$. In the latter case, the models were tested against all samples of consecutive days $d+1, \ldots, d+N$. The prediction quality was evaluated through the root mean squared error in prediction (RMSEP).

For LOD estimation, PLS models were built and tested using a reduced concentration range in which the main LOD assumptions (homoscedastic, normal and unbiased residuals) were satisfied. These assumptions were verified by means of the Levene's test, Saphiro-Wilk test and t-test applied to the residuals of the calibration and intraday test samples. The LOD value was calculated through the linear regression between predicted and experimental concentrations, using only the calibration samples [10].

\section{RESULTS AND DISCUSSION}

The main objective of this section is to present the results of the proposed discontinuous mode and compare its performance to a continuous operating mode. The first experimental day was removed from the dataset because the sensors were not fully stabilized yet. We first computed the RMSEP and LOD as a function of the measurement time. We found that in both groups of sensors the results were stable at the end of the first heating cycle ( $t=25 \mathrm{~s}$.) and adding extra cycles did not significantly improve neither the RMSEP nor the LOD. Hence, a measurement time of $25 \mathrm{~s}$. 


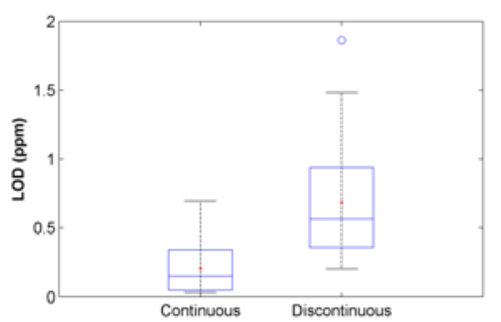

Figure 4. Box plots of the LOD for continuously and discontinuously operated sensors in all experimental days.

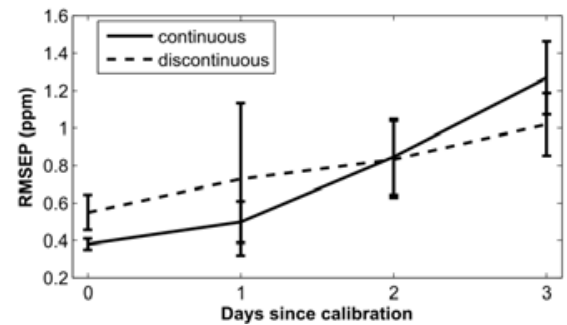

Figure 5. Temporal drift in continuously and discontinuously operated sensors. The plotted lines represent the mean value computed over sensors and experimental days. The error bars indicate the $\mathbf{9 5 \%} \mathrm{t}$-confidence interval for the mean.

was considered optimum and only the results corresponding to that measurement time were reported.

The intraday RMSEP, shown in Fig. 3, measures the error of the calibration model when predictions are made the same day the sensors were calibrated. Results showed that the average error of the discontinuous sensors $(0.5 \mathrm{ppm})$ was moderate in absolute terms but was 1.5 times higher when compared to the continuously operated sensors $(0.32 \mathrm{ppm})$.

For certain applications a more relevant parameter is the LOD. The LOD characterizes the sensor's ability to distinguish between the absence and low concentrations of the target gas. The LOD formulas assume that the residuals resulting from the fitting of calibration concentrations are homoscedastic, normal and unbiased. Because MOX sensors are non-linear devices, the verification of these assumptions is necessary even for calibration ranges as low as $10 \mathrm{ppm}$. For this reason, the LOD was estimated from models calibrated in the range $0-4 \mathrm{ppm}$ in which the assumptions were valid. As it can be seen in Fig. 4, the discontinuous sensors had a mean LOD of $0.7 \mathrm{ppm}$, which is 3.5 times higher than in the group of continuously powered sensors (0.2 ppm) but might be sufficient for most applications.

Because MOX sensors tend to drift over time, especially when there is no continuous power supply, and recalibrations are expensive and time consuming, we explored the performance of both groups of sensors several days after calibration (see Fig. 5). Three days after calibration the RMSEP of the discontinuously operated sensors increased by a factor of 2 with respect to the calibration day. This temporal drift was comparable to the drift seen in the continuously powered sensors, although longer time windows should be explored to confirm the results. Based on these results, the values obtained for the LOD render questionable days after calibration, for both operating modes. Periodic recalibrations might be necessary for applications requiring LOD values close to the metrological LOD of the sensors.

\section{CONCLUSIONS}

This work has shown that MOX sensors can be operated in a discontinuous manner, saving energy up to $60 \%$ compared to the continuous operating mode. We found average intraday RMSEP and LOD values of $0.5 \mathrm{ppm}$ and $0.7 \mathrm{ppm}$, respectively, which were 1.5 and 3.5 times higher than in the continuously operated sensors. Three days after calibration the error increased by a factor of 2 and was comparable in both groups of sensors. The presented approach might be advantageous for applications that do not require continuous and periodic measurements and can tolerate slightly higher prediction errors.

\section{ACKOWLEDGMENTS}

Authors acknowledge TEC2014-59229-R (SIGVOL), BES2015-071698 (SEVERO-OCHOA), TECSPR15-1-0031, and CERCA Programme, 2014-SGR-1445.

\section{REFERENCES}

[1] M. Padilla, et al., Improving the Robustness of Odor Sensing Systems by Multivariate Signal Processing, in: Hum. Olfact. Displ. Interfaces Odor Sens. Present., IGI Global, 2013: pp. 296-316.

[2] A.P. Lee, et al., Temperature modulation in semiconductor gas sensing, Sensors Actuators, B Chem. 60 (1999) 35-42.

[3] G. Korotcenkov, B.K. Cho, Instability of metal oxide-based conductometric gas sensors and approaches to stability improvement (short survey), S\&A B Chem. 156 (2011) 527-538. [4] D. Oletic, V. Jelicic, D. Antolovic, V. Bilas, Energy-efficient atmospheric $\mathrm{CO}$ concentration sensing with on-demand operating MOX gas sensor, in: SENSORS, 2014 IEEE, 2014: pp. 795-798. [5] V. Jelicic, D. Oletic, T. Sever, V. Bilas, Evaluation of mox gas sensor transient response for low-power operation, in: Sensors Appl. Symp. (SAS), 2015 IEEE, 2015: pp. 1-5.

[6] I. Sayhan, et al., Discontinuously Operated Metal Oxide Gas Sensors for Flexible Tag Microlab Applications, (n.d.) 1-5. [7] M. Rossi, D. Brunelli, Ultra low power CH 4 monitoring with wireless sensors, in: SENSORS, 2013 IEEE, 2013: pp. 1-4.

[8] M.M. Macías, et al., Gas sensor measurements during the initial action period of duty-cycling for power saving, Sensors Actuators B Chem. 239 (2017) 1003-1009.

[9] A. Vergara, J.L. Ramírez, E. Llobet, Reducing power consumption via a discontinuous operation of temperaturemodulated micro-hotplate gas sensors: Application to the logistics chain of fruit, Sensors Actuators B Chem. 129 (2008) 311-318

[10] M.. Ortiz, et al., Capability of detection of an analytical

method evaluating false positive and false negative (ISO 11843)

with partial least squares, Chem. Intell. Lab. Syst. 69 (2003) 21-33.

\begin{tabular}{|l|}
\hline Eliminado: Spanish MINECO grants \\
\hline Eliminado: and \\
\hline $\begin{array}{l}\text { Eliminado: . The research group 2014-SGR-1445 receives funding } \\
\text { from }\end{array}$
\end{tabular}

